BULL. AUSTRAL. MATH. SOC.

VOL. $5(1971), 203-204$.

\title{
A skew-Hadamard matrix of order 92 Jennifer Wallis
}

There is a skew-Hadamard matrix of order 92 .

Previously the smallest order for which a skew-Hadamard matrix was not known was 92 . We construct such a matrix below. The orders $<200$ which are now undecided are 100,116, 148, 156, 172, 188, 196; see [2], [3]. The existence of any Hadamard matrix of order 92 was unknown until $1962[1]$.

We construct a skew-Hadamard matrix of Williamson-type by using the matrix

$$
\begin{aligned}
& W=\begin{array}{lllll}
A & B & C & D
\end{array} \\
& \begin{array}{llll}
-B & A & D & -C
\end{array} \\
& \begin{array}{llll}
-C & -D & A & B
\end{array} \\
& \begin{array}{llll}
-D & C & -B & A
\end{array} \text {. }
\end{aligned}
$$

Then if $A$ is a $(1,-1)$ skew-type cyclic matrix of order 23 (that is $a_{i+1, j+1}=a_{i, j}$ where the subscripts are taken modulo 23), B,C,D are $(1,-1)$ anticyclic matrices of order 23 having symmetrical first rows (that is $b_{i, j}=b_{i+1, j-1}, b_{11}=1, b_{1 j}=b_{1,25-j}$ and so on, subscripts modulo 23) and

$$
A A^{T}+B B^{T}+C C^{T}+D D^{T}=92 I_{23}
$$

$W$ is a skew-Hadamard matrix of order 92 .

Suitable first rows for the blocks $A, B, C, D$ are 


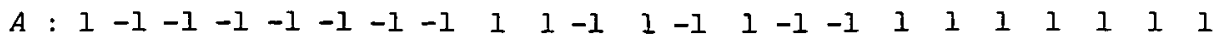

$$
\begin{aligned}
& \begin{array}{llllllllllllllllllllllll}
B & 1 & 1 & -1 & -1 & 1 & -1 & -1 & 1 & 1 & 1 & 1 & -1 & -1 & 1 & 1 & 1 & 1 & -1 & -1 & 1 & -1 & -1 & 1
\end{array} \\
& C: \begin{array}{lllllllllllllllllllllll}
C & 1 & -1 & -1 & -1 & 1 & -1 & 1 & -1 & 1 & -1 & 1 & 1 & -1 & 1 & -1 & 1 & -1 & 1 & -1 & -1 & -1 & 1
\end{array}
\end{aligned}
$$

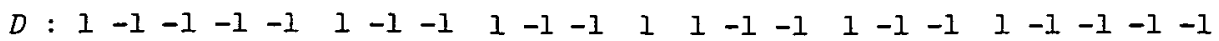

If $W=U+I$ is a skew-Hadamard matrix of order 92 where $I$ is the identity matrix then

$$
\begin{array}{cc}
U+I & U+I \\
U-I & -U+I
\end{array}
$$

is a skew-Hadamard matrix of order 184.

\section{References}

[1] Leonard Baumert, S.W. Golomb and Marshall Hall, Jr, "Discovery of an Hadamard matrix of order 92", Bulz. Amer. Math. Soc. 68 (1962), 237-238.

[2] Jennifer Wallis, " $(v, k, \lambda)$ configurations and Hadamard matrices", J. Austral. Math. Soc. 11 (1970), 297-309.

[3] Albert Leon Whiteman, "An infinite family of skew Hadamard matrices", (to appear).

University of Newcastle, New South Wales. 